\title{
A user survey on the sense of security, Anshin
}

\author{
Yasuhiro Fujihara, Yuko Murayama and Kentarou Yamaguchi
}

\begin{abstract}
Traditional research on security has been based on the assumption that users would feel secure when provided with secure systems and services. In this research we address factors influencing users' sense of security. This paper reports our recent discoveries regarding the structure of the sense of security - Anshin. We conducted a questionnaire survey with one hundred and nine civil servants working for a local government regarding the sense of security. This paper reports our survey.
\end{abstract}

\section{Introduction}

The evaluation of security technology has been concerned with how secure a system is from the theoretical and performance viewpoints. On the other hand, the majority of computer users have not been sure about how secure the systems and services which they use really are. What has been missing is evaluation from users' viewpoints. Not so much work has been done on how well systems and services incorporate users' subjective feelings such as the sense of security. In this research, we try and identify the factors influencing the sense of security.

Throughout this paper, we use a Japanese word for the sense of security, Anshin. Anshin is a Japanese noun which is composed of two words: An and Shin. "An" is to ease, and "Shin" indicates mind. Anshin literally means to ease one's mind [1,2].

According to Yamagishi [3], Anshin is the belief that we have no social uncertainty, whereas trust is needed when we have high social uncertainty. Traditional studies on trust were concerned primarily with cognitive trust, however, Lewis [4]

\footnotetext{
Yasuhiro Fujihara and Yuko Murayama

Faculty of software and Information science, Iwate Prefectural University, 152-52 Sugo, Takizawamura, Iwate 020-0193 JAPAN, e-mail: \{fuji/murayama\} @iwate-pu.ac.jp

Kentarou Yamaguchi

Graduate School of Information Security, Institute of Information Security, 2-14-1 Tsuruya-cho, Kanagawa-ku, Yokohama-shi 221-0085 JAPAN, e-mail: mgs064512@iisec.ac.jp
} 
defined another type of trust, viz. emotional trust. Xiao [5] suggests that the emotional aspect of trust is defined as an emotional security, or feeling secure, or comfortable. $\mathrm{Pu}$ [6] reports that how information was presented affected trust building in user interfaces. Yamazaki and Kikkawa [7] identifies that there is a structure in Anshin through their study on Anshin in epidemic disease.

\section{User survey on Anshin}

We conducted a questionnaire survey on Anshin with about four hundred university students, performing factor analysis on the responses [8]. We identified the six factors contributing to Anshin: security technology, usability, experience, preference, knowledge and assurance. Our previous user survey presented included two types of subjects: those with education in software technology and the others without such an education. The structure of Anshin differs according to whether the subjects know about software technology or not [9]. While the students whose major is Software and Information Science have Anshin based on their knowledge and understanding, those with other majors rely more on Preference and Assurance. This suggests that the two types of the students have different structures of Anshin. The number of subjects without the knowledge was not enough for further analysis. We conducted an additional survey on users without technical knowledge in December 2007. This section describes the previous survey and the new one.

In the future we plan to conduct a larger-scale survey with civil servants. As the survey subjects(civil servants) are somewhat different from the previous ones(students), we conducted an experimental survey. In the current survey, we used a revised questionnaire based on the results from the previous survey. Our new survey includes the following question: "Do you feel that the following thirty four items account for Anshin when you use a service or system through the Internet on a personal use?" Some of the items are listed in Table 1. We used the seven-point Likert scale system ranging from strongly disagree (1) to strongly agree (7), as many such survey have used this scale.

We asked two hundred civil servants working for a local government to answer our questionnaire though e-mail. One hundred and nine civil servants responded via the web. All answers were valid for statistical analysis. All the subjects have basic knowledge of how to operate a computer and they use the Internet on a daily basis. Of the one hundred and nine subjects, seventy one were male, and thirty eight were female, and the average age about 39.3.

\section{Factor Analysis results}

We analyzed the current survey data using explanatory factor analysis (EFA). In this study, we examined the factors of Anshin that existed behind between question- 
naire items, by using EFA. The main results were as follows: EFA using maximumlikelihood method and promax rotation found that seven factors are present. We tried the analysis several times to derive the effective items out of thirty four and found that the twenty eight items would be feasible as contributing to Anshin. All items have factor loading above 0.581 . The seven factors explained by $73.13 \%$ of the total variance.

We present a brief summary of each factor. Each factor is composed of multiple questionnaire entries. Following items contained in each factor, which is represented in descending order of factor loading.

Factor 1: Security Technology consists of six items (Q15, Q08, Q14, Q07, Q13, Q04, Q01) about security technology. Most items indicate measures for security such as protection of personal data.

Factor 2: Usability consists of five items (Q26, Q24, Q25, Q23, Q27) items about satisfaction with the user interface (UI). Especially, it has subjective assessment of the quality of UI; for example, usability, attractive design and user-friendliness.

Factor 3: Preference for Interface consists of three items (Q21, Q22, Q20) about preference for interface design. In other words, it shows the user's likes and tastes on interface.

Factor 4: Knowledge consists of four items (Q18, Q19, Q16, Q17) about knowledge of information technology. It shows the perception of risk, and understanding of risk or threat based on a user's prior knowledge.

Table 1 Item details

Factor Items
Factor 1 Q15 Companies care about security.
Q08 Personal information which I input is managed carefully and it will not be leaked
to the outside.
Q14 I feel secure when I use the system/service.
easy to use the system/service.
Q24 The usability of the system is excellent.
Q25 Since the system/service provides deliberate explanation on how to use it, I get the
impression that I am treated well.
Q22 The layout and color of the system design are attractive.
Q20 I feel familiar about the system design.
Factor 3 Q21 The system design is attractive.
Q19 I know the risks and security threats when I use the system/service.
Factor I know something about the mechanism of security tools.
Q31 I feel secure without any specific reason when I use the system/service.
Q29 Since I frequently use the system/service, I am not worried about its security.
Factor 5 Q32 I like the system/service without any specific reason.
Q03 I am confident in the competence of the provider and its owner.
Q12 Even if I had a problem, the system would assist me to solve it.
Q11 Even if I had a problem, it would be fixed when the system restarts.
Factor 6 Q2 The service provider and its owner company have the confidence of society.
Factor 7 Q10 Even if I had a trouble, I would be protected by a guarantee.


Factor 5: Intuitive Impression consist of three items (Q32, Q31, Q29) about impression based on intuition. It is a fully subjective factor such as user's experience, user's preference of the service, and recommendation of one's family and friends.

Factor 6: Assurance consists of three items (Q02, Q06, Q03) concerned with how much confidence the user feels in society and the user's expectation ability of the others, security, safety, and so forth.

Factor 7: Action on a trouble consists of three items (Q10, Q12, Q11) about expected action of the system on a trouble. In other words, it shows the system would assist the user to solve the problem or the problem would be fixed when the system restarts.

\section{Discussion}

Based on the current survey, the seven factors including the newly found two factors of Intuitive Impression and Action on a trouble have been found. The items belonging to Intuitive Impression were composed of user experience, preference, impression and so on. The factor of Intuitive Impression included the items that made up the Experience factor in the previous survey. While Action on a trouble was included in the factors of Information security in the previous survey, it was found as an independent factor this time.

We asked the subjects a question about knowledge of information security on current survey. We classified the subjects in three groups of "those who know in details" (seventeen people), "those who know like everyone else" (fifty people), "those who do not know" (forty two people), compared with ordinary people. In order to study the relationship between the knowledge of information security and each respective factor, we made an analysis of variance that made the knowledge of information security as a factor. As a result of analysis, there are significant differences only in the usability factor $(\mathrm{F}(2,108)=3.710, \mathrm{p}<.05)$, and the intuitive impression factor $(\mathrm{F}(2,108)=3.163, \mathrm{p}<.05)$. That is, those who do not have information security knowledge, tended to understand the degree of security based on usability and intuitive impression. Accordingly it indicates that the structure of Anshin is different according to the knowledge of information security.

\section{Conclusions}

We conducted a user survey with civil servants, concerning Anshin in information security. We had one hundred and nine cases of data used in this analysis, which is not quite a sufficient number to make a detailed analysis. As the current survey was a preliminary experiment to prepare for a larger-scale survey, we cannot lead to a conclusion from the results of this survey. Some items in the questionnaire were hard to answer, according to users who do not know much about information security. In 
the future, we plan to improve the questionnaire and conduct a user survey with a greater number of subjects for various groups, finding out the structure of Anshin in information security.

Acknowledgements Special thanks to Natsuko Hikage of Nippon Telegraph and Telephone, and Dr. Carl Hauser of Washington State University. Without their help this research was not possible. We thank Ginny Hauser for her assistance with our translation of our questionnaire into English.

\section{References}

1. Murayama, Y., Hikage, N., Hauser, C., Chakraborty, B. and Segawa, N. (2006) An Anshin Model for the Evaluation of the Sense of Security, Proc. of the 39th Hawaii International Conference on System Science (HICSS'06), Vol.8:205a

2. Hikage, N., Murayama, Y. and Hauser, C. (2007) Exploratory survey on an evaluation model for a sense of security, Proc. of the 22nd IFIP TC-11 International Information Security Conference (SEC2007):121-132

3. Yamagishi, T. (1998) The structure of trust: The evolutionary games of mind and society, Tokyo University Press. English version is available at : http://lynx.let.hokudai.ac.jp/members/yamagishi/english/ (Cited 14 Jan 2008)

4. Lewis, J. D. and Weigert, A. (1985) Trust as a Social Reality, Social Forces, Vol.63, No.4:967985

5. Xiao, S. and Benbasat, I. (2003) The formation of trust and distrust in recommendation agents in repeated interactions: a process-tracing analysis, Proc. of the 5th international conference on Electronic commerce (ICEC'03):287-293

6. Pu, P. and Chen, L. (2006) Trust building with explanation interfaces, Proc. of the 11th international conference on Intelligent user interfaces (IUI'06):93-100.

7. Yamazaki, M. and Kikkawa, T. (2006) The Structure of Anxiety Associated with Avian Influenza and Pandemic Influenza, the 47th annual conference of the Japanese Society of Social Psychology:676-677(in Japanese)

8. Hikage, N., Hauser, C. and Murayama, Y. (2007) A statistical discussion of the sense of security, Anshin, Information Processing Society of Japan (IPSJ) Journal, Vol.48, No.9:31933203(in Japanese)

9. Murayama, Y., Hikage, N., Fujihara Y. and Hauser C. (2007) The structure of the sense of security, Anshin, 2nd International Workshop on Critical Information Infrastructures Security:85-96 